\title{
Corrections
}

\section{Correction: Liu et al., "Vectored Intracerebral Immunization with the Anti-Tau Monoclonal Antibody PHF1 Markedly Reduces Tau Pathology in Mutant Tau Transgenic Mice"}

In the article "Vectored Intracerebral Immunization with the Anti-Tau Monoclonal Antibody PHF1 Markedly Reduces Tau Pathology in Mutant Tau Transgenic Mice" by Wencheng Liu, Lingzhi Zhao, Brittany Blackman, Mayur Pamar, Man Ying Wong, Thomas Woo, Fangmin Yu, Dolan Sondhi, Stephen M. Kaminsky, Ronald G. Crystal, and Steven M. Paul, which appeared on pages 12425-12435 of the December 7, 2016 issue, author Maria J. Chiuchiolo was inadvertently omitted from the author list. The authors apologize for the oversight. The corrected author list is as follows: Wencheng Liu, Lingzhi Zhao, Brittany Blackman, Mayur Pamar, Man Ying Wong, Thomas Woo, Fangmin Yu, Maria J. Chiuchiolo, Dolan Sondhi, Stephen M. Kaminsky, Ronald G. Crystal, and Steven M. Paul Her affiliation is as follows: Department of Genetic Medicine, Weill Cornell Medicine, New York, New York, 10021. The Author Contributions footnote should have read "Author contributions: W.L. and S.M.P. designed research; W.L., L.Z., M.J.C., B.B., M.P., M.Y.W., T.W., and F.Y. performed research; W.L., M.J.C., D.S., S.M.K., R.G.C., and S.M.P. contributed unpublished reagents/analytic tools; W.L. and S.M.P. analyzed data; and W.L. and S.M.P. wrote the paper." This correction does not affect the conclusions of the paper.

DOI: $10.1523 / J N E U R O S C I .0488-17.2017$

\section{Correction: Favero and Castro-Alamancos, "Synaptic Cooperativity Regulates Persistent Network Activity in Neocortex"}

In the article "Synaptic Cooperativity Regulates Persistent Network Activity in Neocortex" by Morgana Favero and Manuel A. Castro-Alamancos, which appeared on pages 3151-3163 of the February 13, 2013 issue, the authors regret a typographical error. Everywhere in the article where it mentions the AMPA-receptor antagonist “GYKI35348”, it should say "GYKI53655.” This correction does not affect the results or conclusions of the paper.

DOI: 10.1523/JNEUROSCI.0642-17.2017 